\title{
Comparison between subtraction and dynamic MRI in assessing treatment response following radiofrequency ablation in patients with hepatocellular carcinoma
}

\author{
Ahmed Elshenawy Gabr, Hisham S. Wahba Mikhael and Samar M. El-Maadawy* (1)
}

\begin{abstract}
Background: Hepatocellular carcinoma (HCC) is one of the most prevalent cancers worldwide, and if left untreated, one of the most lethal. Ablative therapies including radiofrequency ablation (RFA) play increasingly important role for patients with liver tumors who are not surgical candidates. Monitoring treatment response following ablation is crucial in oncologic imaging. Dynamic contrast-enhanced MRI can assess changes in tumor vascularity and perfusion while subtraction imaging is useful in differentiating residual tumor from post-ablation parenchymal changes. The aim of this study is to compare the role of subtraction MRI and conventional dynamic MRI in assessing treatment response following RFA in patients with HCC.

Results: The study included 48 patients with 62 HCC lesions who underwent RFA from May to October 2020, followed by MRI evaluation with 1-month interval. Two readers with experience in hepatic imaging interpreted the dynamic and subtraction dynamic MRI. The hepatic focal lesions were classified into "well-ablated" and "residual" groups according to MRI findings, and the agreement between the two readers was evaluated. Using dynamic MRI, the first reader reported 38 well-ablated lesions, and the second reader agreed in 34 of them (89.5\%). Residual disease was reported by the first reader in 22 lesions and the second reader disagreed in 10 of them (45.5\%) where complete ablation was reported. Thirty-eight out 44 well-ablated lesions (86.4\%) showed high signal intensity on non-enhanced $\mathrm{T} 1$ images, and 28 lesion (63.6\%) showed intermediate T2 signal. All the mis-matched readings occurred in lesions with a high signal intensity in pre-contrast T1 images. Moderate agreement between the two readers was found with Kappa value of 0.467 . Significant additive value of subtraction technique to dynamic MRI was detected with a $P$ value of 0.009. No major complications recorded except for a single case of major portal vein branch occlusion.
\end{abstract}

Conclusion: MRI is a powerful imaging tool in assessing tumor viability and complications after RFA in patients with HCC. Dynamic MRI study is the gold standard in detecting recurrent lesions while subtraction technique is crucial in differentiating between arterial enhancement due to residual disease and normal hyperintense T1 signal of the ablation zone.

Keywords: Subtraction MRI, Dynamic MRI, Hepatocellular carcinoma, Radiofrequency ablation

*Correspondence: samarmaadawy@gmail.com

Department of Radiology, National Cancer Institute, Cairo University, Cairo, Egypt

\section{Background}

Hepatocellular carcinoma $(\mathrm{HCC})$ is one of the most frequent malignancies in the world, and if left untreated, it has a dismal prognosis [1]. HCC treatment is complicated, and depending on the stage of the disease, various 
therapies may be accessible to the same patient [2]. Surgical resection is considered the gold standard therapy for HCC. However, due diffuse distribution of the tumor and the severity of underlying cirrhosis, only one out of 10-15 patients are eligible for surgery [3]. Following technical advancements, ablative therapies currently play an increasingly important role for patients with liver tumors who are not candidates for surgery [4].

Ablative methods can be classified into either chemical or thermal. Chemical ablation employs acetic acid and ethanol, whereas thermal ablation uses either cold (cryoablation) or heat (radiofrequency ablation RFA, microwave ablation and laser ablation) [5]. RFA is considered the most commonly utilized ablative method for both primary and secondary hepatic tumors. Irrespective of the ablative technique, coagulative necrosis almost inevitably occurs which produces similar imaging characteristics during follow-up studies [1].

Post-ablative imaging assessment is of utmost importance for the detection of residual or recurrent tumors as well as post-treatment complications [1]. Contrastenhanced imaging techniques in the form of contrastenhanced ultrasound (CEUS), computed tomography $(\mathrm{CT})$, and magnetic resonance imaging (MRI) are the most widely used imaging tools for post-ablation assessment [4]. Previous studies have suggested that MRI is more accurate in the detection of residual or recurrent tumors in the ablative bed [6-8].

The European Association for the Study of Liver Disease (EASL) has recommended the use of lesions' enhancement pattern rather than the interval size change as the preferred method for determining response to treatment [9]. However, post-ablation reactive granulation tissue may result in homogeneous rim augmentation. Therefore, diffusion MRI may offer additional benefits to assess tumor necrosis [10]. Following RFA, diffusionweighted imaging (DWI) has shown good results in the assessment residual or recurrence at the ablation site particularly when it is combined with conventional dynamic contrast-enhanced imaging [10]. Perfusion MRI also appears to be a promising technique in the evaluation of HCC in cirrhotic patients. The application of quantitative vascular measurements can aid in the assessment of RFA efficacy [11]. Subtraction dynamic MRI imaging, where the unenhanced T1-weighted images are subtracted from identical images after gadolinium injection, can potentially offer additional post-RFA evaluation since the remaining signal in the subtracted images will be only due to enhancement indicating tumor residual or recurrence [12].

The aim of the present study is to compare the role of subtraction MRI and conventional dynamic MRI in the assessment of treatment response following RFA in patients with HCC. A proposed refined technique and a standardized MRI protocol after HCC RFA will also be described.

\section{Methods}

This prospective single institution study included all consecutive patients diagnosed with HCC who were referred to the Radiology Department for RFA followed by MRI assessment from May 2020 to October 2020. The study was approved by our institution Ethics Committee. A written informed consent was obtained from all patients who agreed to take part in this study.

Inclusion criteria included inoperable HCC patients with liver cirrhosis related to chronic viral hepatitis or who are not surgical candidates due to anesthesia contraindications, advanced age or the presence of other comorbidities. Patients should have fewer than five tumors, each of which not more than $5 \mathrm{~cm}$ with no radiological evidence of extrahepatic disease. Exclusion criteria included tumors other than HCC, excessive tumor burden within the liver, location of the tumor close to vital structures like the diaphragm, liver surface, gall bladder, major bile duct or blood vessel as well as MRI contraindications which included patients with metallic implants, cardiac pacemakers, MRI-incompatible prosthetic cardiac valves, and claustrophobic patients. All patients underwent a comprehensive clinical evaluation as well as a review of past laboratory and radiological tests prior to RFA and MRI exams.

\section{Radiofrequency ablation technique}

The Cool-tip ${ }^{\text {TM }}$ Radiofrequency Ablation System E Series (Covidien AG) was used in our study which consists of three primary components: the ablation needle, electric generator, and the grounding pads. During the RFA procedure, an ablation needle is inserted into the long axis of the hepatic focal lesion under ultrasound guidance (GE Healthcare Logic E9) using C2-9-D 2-9 MHz broad-spectrum convex probe. From the needle tip, the electrodes are inserted into the target hepatic focal lesion from the needle tip resembling the shape of an umbrella using a plunger on the needle hub. The generator is started which produces $200 \mathrm{~W}$ power in a pulsed fashion, the target temperature is selected from 50 to $100{ }^{\circ} \mathrm{C}$, and the grounding pads are placed on the patient thigh. Once the target temperature is reached, the heat kills the target tissue. When the temperature of a tissue rises above 50 degrees Celsius, cell protein is permanently destroyed, and coagulation necrosis begins. Cell death happens very instantaneously at temperatures above $60{ }^{\circ} \mathrm{C}$. It takes about $15-30 \mathrm{~min}$ to perform a $3-$ to $5-\mathrm{cm}$ hepatic focal lesion ablation. The treatment is monitored by ultrasound and the ablated tissue appears as a progressively 
enlarging focal lesion of increased echogenicity due to release of gas bubbles. The ablation zone must cover the entire tumor with a 5-mm safety margin. In case of large focal lesions, multiple overlapping ablations were performed to ensure full ablation.

\section{MRI protocol}

All patients were scheduled for MRI 1 month after the ablation. All MRI examinations were performed on a 1.5 T MRI scanner (Philips Achieva, Koninklijke Philips N.V., Netherlands).

\section{Pre-contrast imaging (Table 1)}

\section{Dynamic study}

Dynamic study was performed after bolus injection of $0.1 \mathrm{mmol} / \mathrm{kg}$ body weight of Gd-DTPA using automatic injector (Medrad, Spectris Solaris, EP MR injection system).

Dynamic imaging using 3D fat-suppressed T1-weighted gradient echo sequence (THRIVE; T1 highresolution isotropic volume examination). The dynamic series comprised one pre-contrast series followed by four post-contrast series which included early and late arterial, and portal phases with 19-21 s intervals (17 s for image acquisition with breath-holding and 2-4 s for rebreathing) and a 5-min delayed phase imaging. To minimize the possibility of image misregistration, all patients in our study were imaged in end expiration.

Acquisition parameters for 1.5 Tesla machines were TR $10 \mathrm{~ms}$, TE $4.6 \mathrm{~ms}$, flip angle $15^{\circ}$, matrix size, $172 \times 163$, field of view $300-350 \mathrm{~mm}$ and slice thickness $7 \mathrm{~mm}$.

\section{Analysis of MRI images}

Images were transferred to (Phillips Extended MR Workspace) workstation for image processing. Each lesion morphological characteristics such as size, margins and signal intensity at T1, T2 and SPAIR (spectral attenuated inversion recovery) images were recorded.

Post-ablation residual or recurrent tumor viability and complications were assessed.

Table 1 Parameters for hepatic MR imaging

\begin{tabular}{llllll}
\hline Sequence & TR $(\mathbf{m s})$ & TE $(\mathbf{m s})$ & FOV $(\mathbf{m m})$ & Flip angle & $\begin{array}{l}\text { Slice } \\
\text { thickness } \\
(\mathbf{m m})\end{array}$ \\
\hline Axial T1 TFE & 10 & 4.6 & $300-350$ & 15 & 7 \\
Axial T2 TSE & 1000 & 80 & $300-350$ & 90 & 7 \\
Axial TS SPAIR & 1000 & 80 & $300-350$ & 90 & 7 \\
\hline
\end{tabular}

TR repetition time, TE time to echo, FOV field of view, TFE turbo field echo, TSE turbo spin echo, SPAIR spectral attenuated inversion recovery

\section{Analysis of dynamic study}

After the single pre-contrast series, we performed early and late arterial, portal, and delayed phases. The pattern of enhancement seen through the sequential dynamic series was then analyzed.

\section{Subtraction dynamic study analysis}

Subtraction imaging, which is an automated method available on the MRI workstation was performed. The unenhanced T1-weighted sequence was digitally subtracted from an identical sequence taken after gadolinium injection.

The pre-contrast series was digitally subtracted from the late arterial phase in the dynamic study. The residual signal on the subtracted images is only attributable to enhancement because the native T1 signal has been deleted. Enhancement patterns in the subtraction dynamic imaging were assessed.

\section{Interpretation of $\mathbf{M R I}$ image}

Ablation zone signal at T1-, T2- and SPAIR-weighted images was classified as low, high or heterogenous.

Two readers with more than 10 years' experience in the field of hepatic imaging interpreted the images. The first reader interpreted the dynamic MRI and was blinded to the subtraction MRI images; the second reader interpreted both dynamic and subtraction MRI images. Each reader had access to the patients' clinical details and assessed the MRI images separately.

\section{Dynamic study interpretation by the first reader}

Enhancement in the arterial phase with delayed wash out were recorded suggesting residual tumor viability.

Absence of arterial phase enhancement suggested good tumor ablation.

\section{Dynamic and subtraction dynamic studies interpretation by the second reader}

The second reader-who was blinded to the first reader findings - also interpreted the dynamic study to detect arterial phase enhancing lesions that suggested residual tumor activity.

Contrast wash out. Reduction in the enhancement on delayed phase compared with early phase dynamic imaging.

Subtraction images were used to confirm/correct the recorded findings, demonstrating that the high signal in the arterial phase is attributable to enhancement rather 
than the original pre-contrast high $\mathrm{T} 1$ signal of coagulative necrosis.

\section{Rim enhancement of the ablation zone could be either} Granulation tissue rim: delayed or persistent phase enhancement.

Nodular enhancement: suggests tumor recurrence.

\section{Pattern of enhancement impressive for tumor progression}

The classification reported by Chopro et al. [13] were followed as follows:

"Halo type": thick irregular rim of enhancement surrounding the ablation zone.

"Nodular type": foci of nodular enhancement at ablation zone margins.

"Gross enlargement type": increase in the size of the ablation zone.

\section{The patients were classified into two groups}

Well-ablated (Resolved) group: both readers found no evidence residual or recurrent tumor viability on MRI (regardless of the presence of newly developed lesions).

Residual (Unresolved) group: both readers reported signs of recurrent or residual tumor.

\section{Standard of reference}

Since most patients who perform RFA do not undergo surgery, pathologic confirmation was difficult to obtain. Furthermore, because recurrent lesions are generally small nodules, biopsy may result in sampling error. Therefore, we considered the following:

Well-ablated (resolved) lesions were considered by the absence of enhancement on both the arterial phase of the dynamic study and the subtraction dynamic study.

Residual tumor activity (unresolved) lesions were suggested by the presence of enhancement on arterial phase and wash out of contrast on delayed phase as well as positive enhancement on the subtracted images or a focal area at the margin of the ablation zone that shows early, or late arterial phase enhancement proved by subtraction images or contrast wash out in the delayed phase. In case of disagreement between the first and second readers, a conjoint meeting to reach a consensus was done using subtraction MRI as the gold standard.

\section{Statistical methods and analysis}

Data were analyzed using IBM SPSS advanced statistics version 20 (SPSS Inc., Chicago, IL). Numerical data were expressed as mean and standard deviation or median and range as appropriate. Qualitative data were expressed as frequency and percentage. Chi-square test or Fisher's exact test was used to compare between well-ablated and unresolved lesions based on subtraction MRI. Kappa test was used to evaluate agreement between two diagnostic methods using subtraction as the gold standard. All tests were two-tailed. A $P$ value $\leq 0.05$ was considered significant and $<0.01$ was considered highly significant. Kappa values from 0.40 to 0.59 are considered moderate, 0.600.79 substantial, and 0.80 outstanding.

\section{Results}

After exclusion criteria, our study included 48 patients with 62 hepatic focal lesions. Thirty-eight patients (79.2\%) were males, whereas ten $(20.8 \%)$ were females. The age ranged from 44 to 71 years with a mean of 53.1 years. The hepatic focal lesions were classified into two groups by two readers. Resolved (well-ablated) group showed no MRI signs of residual or recurrent viability at the ablated lesion regardless of the presence of other focal hepatic lesions, while unresolved (residual) group showed evidence of residual or recurrent viability. Dynamic MRI imaging interpretation by the first reader revealed that 40 lesions (64.5\%) were well-ablated, while 22 lesions (35.5\%) showed residual viability. The subtraction dynamic MRI imaging interpretation by the second reader revealed that 44 lesions $(71.0 \%)$ were ablated, 16 lesions $(25.8 \%)$ showed residual viability, and two lesions (3.2\%) were mis-registered. Figures 1, 2, 3 and 4 show examples of cases encountered in our study.

The signal intensity of the ablated zone in the nonenhanced T1-weighted images was classified into three types: heterogeneous high signal, high signal, and isointense signal. Fifty out of 62 ablated lesions (80.6\%) showed homogeneous very high signal intensity, eight lesions $(12.9 \%)$ showed heterogeneous signal and four lesions $(6.5 \%)$ showed iso-intense signal.

The signal intensity of the ablation zone in the T2 images was classified into three types: heterogeneous low signal, homogeneous low signal, and intermediate signal. Thirty-eight out of 62 lesions (61.3\%) showed intermediate signal, 20 lesions (32.3\%) showed homogeneous low signal, and four lesions (6.5\%) showed heterogenous low signal.

Correlation between the non-enhanced T1-weighted images and T2 signal intensity in the ablation zone with residual tumor activity was performed according to the second reader subtraction dynamic MRI interpretation after excluding the two mis-registered lesions as shown in Table 2.

As given in Table 2, 38 out 44 well-ablated lesions (86.4\%) showed high signal intensity on non-enhanced T1 images, and 28 lesion (63.6\%) showed intermediate T2 signal. On the other hand, ten out of 16 lesion showing residual disease (62.5\%) showed high T1 signal and half of them showed intermediate T2 signal. No statistically significant correlation between $\mathrm{T} 1$ and $\mathrm{T} 2$ signal 

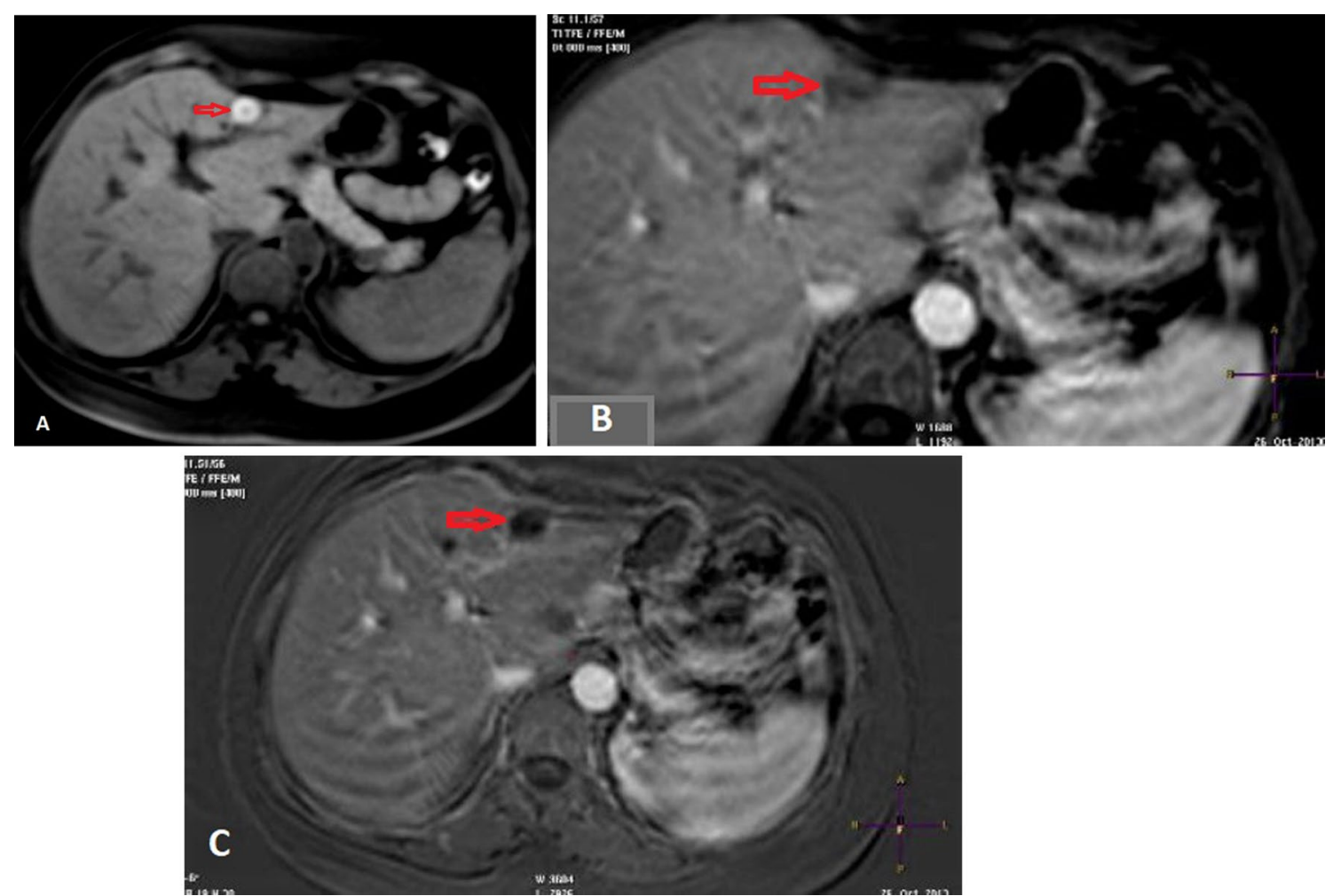

Fig. 1 A 63-year-old female patient with liver cirrhosis underwent radiofrequency ablation of segment II hepatic lobe focal lesion. A, B, and C corresponding axial T1 pre-contrast, dynamic MR arterial phase and subtraction images. A The targeted left lobe focal lesion (open red arrow) has high signal on the T1 pre-contrast. B The corresponding arterial phase shows hazy mixed signal within the targeted lesion (open red arrow). C The subtraction image shows that the entire lesion is hypointense (open red arrow) with no evidence of enhancing nodules

(See figure on next page.)

Fig. 2 A 60-year-old male patient with hepatocellular carcinoma on top of liver cirrhosis underwent radiofrequency ablation of two left hepatic lobe focal lesions. A Axial T1- pre-contrast image shows two heterogeneous but mostly hyperintense left lobe hepatic focal lesions (open yellow and red arrows). B, C Axial dynamic MR arterial and delayed phases show the smaller hepatic focal lesion (open yellow arrow) is hyperintense in $\mathrm{T} 1$ and arterial phase with relative washout in the delayed phase. The other focal lesion (open red arrow) showed suspected enhancing nodule with wash out in the delayed phase. D Subtraction image of the smaller focal lesion (open yellow arrow) showed no evidence of enhancing viable tumoral tissue. The subtraction of the larger focal lesion (open red arrow) on the other hand confirms enhancing viable nodule seen at about 6-7 o'clock position. $\mathbf{E}$ The color map images of the mural residual nodule shows enhancement relative to the nearby ablated zone (open red arrow). $\mathbf{F}$ Enhancement curve (time-signal intensity) of the residual mural nodule shows arterial enhancement with rapid wash out

intensity and neoplastic viability was detected with a $P$ value of 0.648 and 0.678 , respectively.

Correlation between the MRI imaging findings of the first reader (dynamic MRI) and second reader (subtraction dynamic MRI) is shown in Table 3.

As shown in Table 3, using dynamic MRI, the first reader reported 38 well-ablated lesions, and the second reader agreed with the first reader in 34 of them (89.5\%). Residual disease was reported by the first reader in 22 lesions and the second reader disagreed in 10 of them $(45.5 \%)$ where complete ablation was reported.
Evaluation of the 14 lesions where there was disagreement between the first and second readers was done by a conjoint meeting to reach a consensus using subtraction MRI as the gold standard. It was found that all the mismatched readings occurred in lesions having high signal intensity in the pre-contrast T1 images. Moderate agreement between the two readers was found with a Kappa value of 0.467 . Significant additive value of subtraction technique to dynamic MRI was also detected with a $P$ value of 0.009 .

All patients in our study tolerated the procedure well. No major complications were recorded in our study 

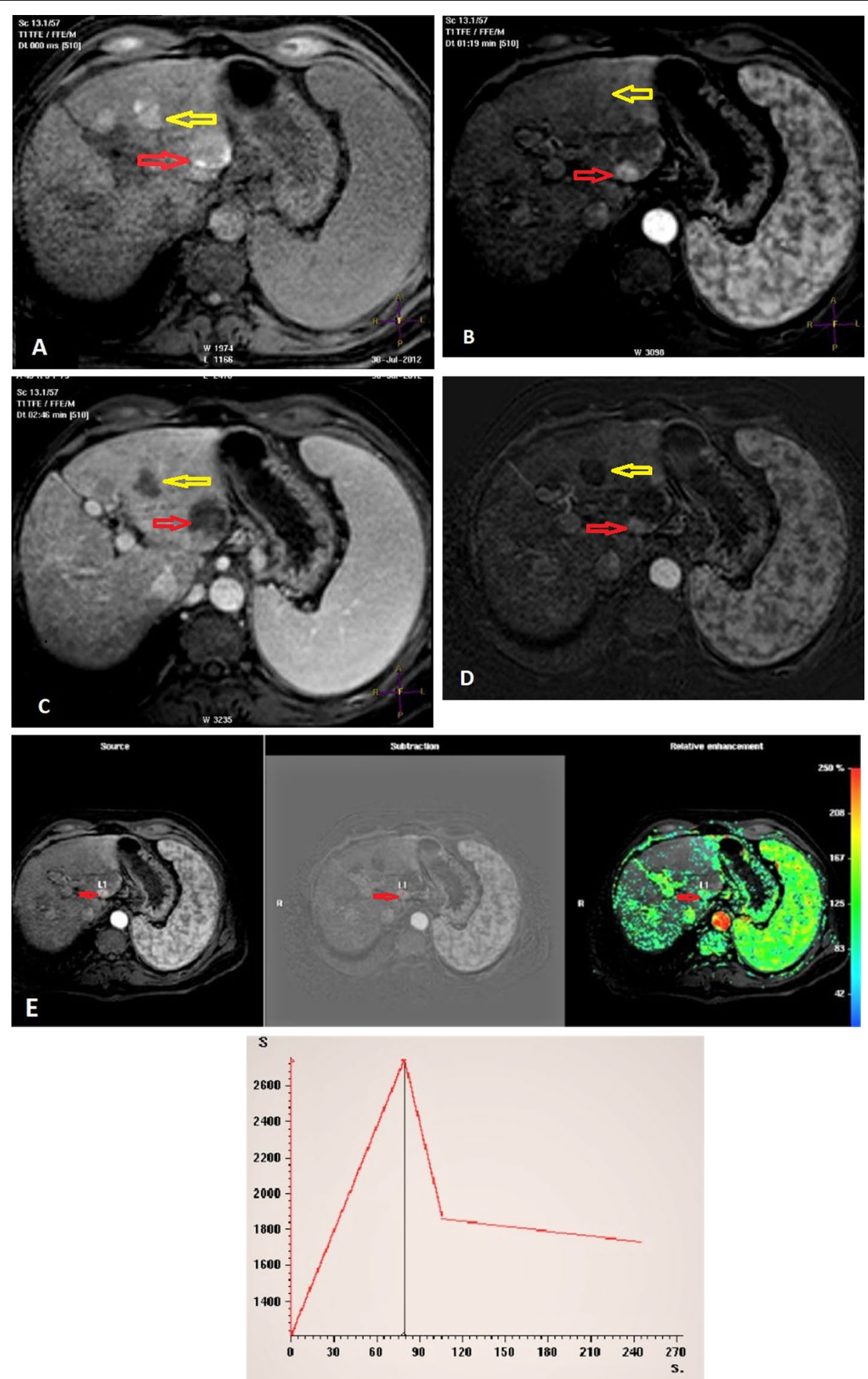

Current dynamic

Reference dynamic

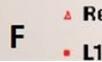

Fig. 2 (See legend on previous page.) 

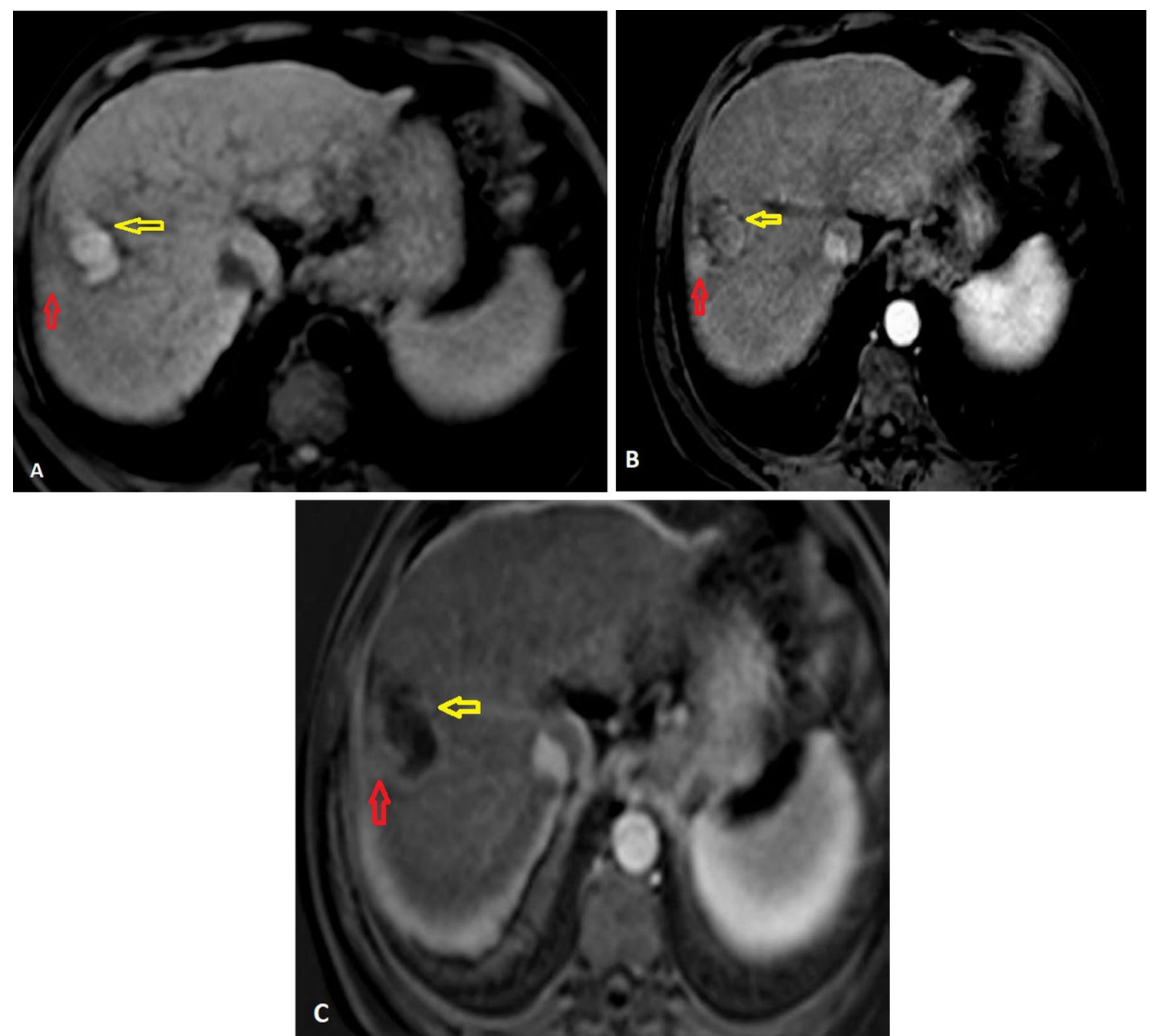

Fig. 3 A 65-year-old male patient with liver cirrhosis underwent radiofrequency ablation of right hepatic lobe focal lesion. A Axial T1 pre-contrast image shows heterogeneous but mostly hyperintense focal lesion (open yellow arrow) with iso-intense portion (open red arrow). B Axial dynamic arterial phase shows hyperintense focal lesion which could be attributed to enhancement or residual disease (open yellow arrow) with suspected enhancing nodule seen at about 7 o'clock position (open red arrow). C Subtracted image shows conspicuous area of nodular enhancement, consistent with residual tumoral activity (open red arrow) with subtraction of the high signal seen in the arterial phase (open yellow arrow) therefore suggestive of coagulative necrosis rather than enhancement of residual disease

except for a single case of occlusion of major portal vein branch.

\section{Discussion}

In the present study, we evaluated the role of subtraction dynamic MRI in the assessment of treatment response after RFA in patients with HCC. According to our findings, subtraction MRI is a useful problem-solving tool for detecting residual or recurrent tumors as well as any post-procedure complications that can aid in a confident diagnosis.
The study demonstrated that $80.6 \%$ of the hepatic focal lesions exhibited homogeneously very high signals in the non-enhanced T1-weighted images which can be explained by the coagulative hemorrhagic necrosis caused by RFA thermal effect. Our findings were in agreement with previous investigators who examined 50 hepatic focal lesions treated with RFA half of which showed a very high T1 signal [14]. Similar observations were also reported by other investigators with the majority of their ablated hepatic focal lesions eliciting heterogeneous high signal in T1-weighted images [12, 15-18]. Correlation between $\mathrm{T} 1$ signal intensity and the ablation 

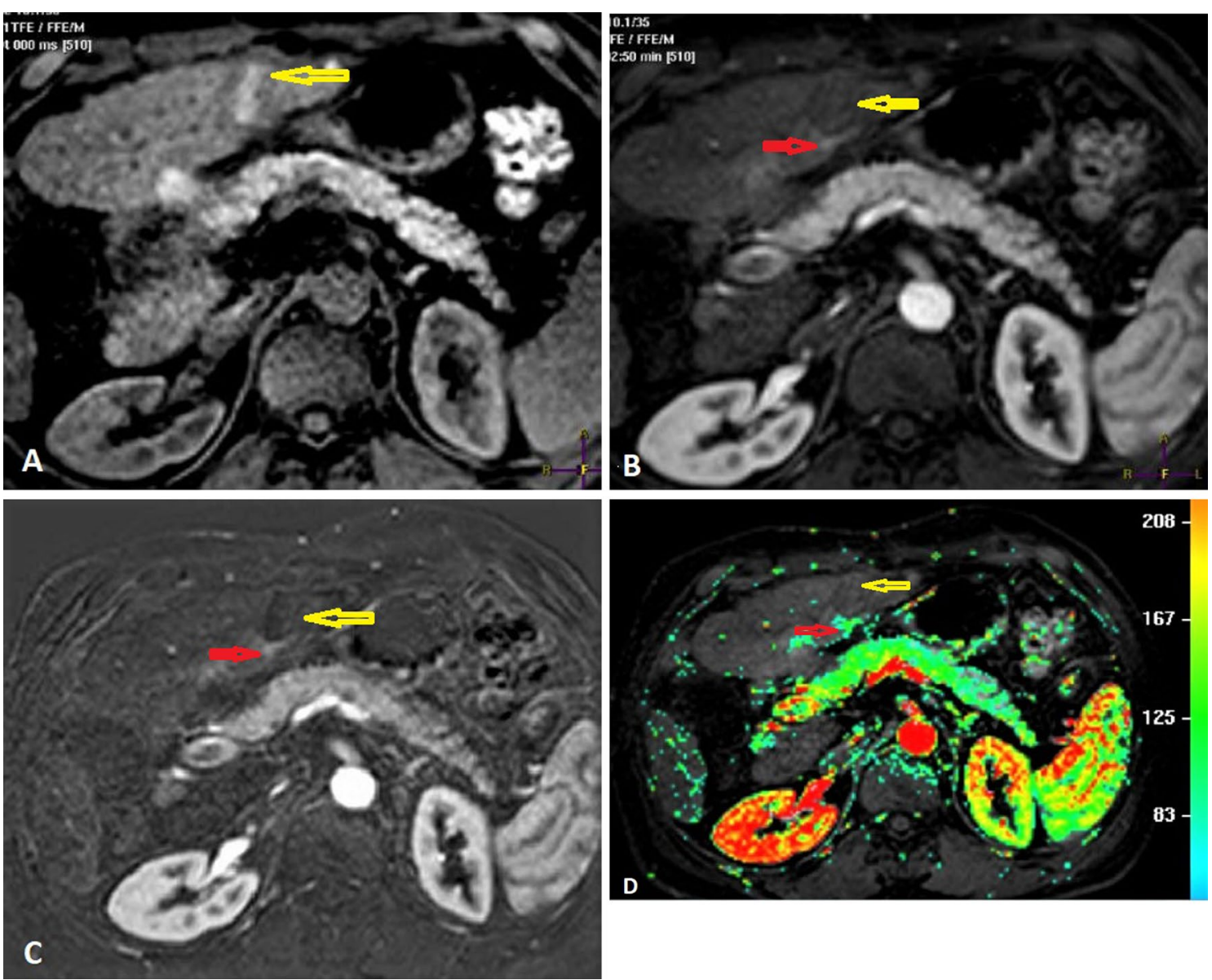

Fig. 4 A 66-year-old male patient with liver cirrhosis underwent radiofrequency ablation of left hepatic lobe focal lesion. A Axial T1 pre-contrast image showing hyperintense left hepatic lobe focal lesion after radio frequency ablation (open yellow arrow). B Suspected residual nodule seen at about 6 o'clock position in the arterial phase (open red arrow). C The subtracted images shows that that suspected residual nodule (open red arrow) is hyperintense denoting enhancing residual disease. The remaining portion of the nodule is hypointense denoting complete ablation. $\mathbf{D}$ Color-coded perfusion map shows enhancement of the residual nodule (open red arrow) with non-enhancing ablation zone (open yellow arrow)

Table $2 \mathrm{~T} 1$ and T2 signal distribution characteristics in unresolved and resolved lesions based on subtraction MRI findings

\begin{tabular}{llll}
\hline MRI images & \multicolumn{2}{l}{ Subtraction dynamic MRI $(\boldsymbol{n}=\mathbf{6 0})$} & P value \\
\cline { 2 - 3 } & $\begin{array}{l}\text { Well-ablated } \\
\boldsymbol{n = 4 4}(\%)\end{array}$ & $\begin{array}{l}\text { Residual } \\
\boldsymbol{n}=\mathbf{1 6}(\%)\end{array}$ & \\
\hline $\begin{array}{l}\text { Non-enhanced T1 signal intensity } \\
\text { High }\end{array}$ & $38(86.4)$ & $10(62.5)$ & 0.648 \\
Heterogeneous & $2(4.5)$ & $6(37.5)$ & \\
Iso-intense & $4(9.1)$ & $0(0)$ & 0.678 \\
T2 signal intensity & & & \\
Intermediate & $28(63.6)$ & $8(50.0)$ & \\
Low & $14(31.8)$ & $6(37.5)$ & \\
Heterogeneous & $2(4.5)$ & $2(12.5)$ & \\
\hline
\end{tabular}

Table 3 Correlation between conventional dynamic MRI and subtraction dynamic MRI as the gold standard

\begin{tabular}{|c|c|c|c|c|c|}
\hline & \multicolumn{4}{|c|}{ Subtraction dynamic MRI $(n=60)$} & \multirow[t]{3}{*}{ Total } \\
\hline & \multicolumn{2}{|c|}{$\begin{array}{l}\text { Well-ablated } \\
n=44\end{array}$} & \multicolumn{2}{|c|}{$\begin{array}{l}\text { Residual } \\
n=16\end{array}$} & \\
\hline & $n$ & $\%$ & $n$ & $\%$ & \\
\hline \multicolumn{6}{|l|}{ Dynamic MRI } \\
\hline Well-ablated & 34 & 77.3 & 4 & 25.0 & 38 \\
\hline Residual & 10 & 22.7 & 12 & 75.0 & 22 \\
\hline
\end{tabular}

zone and residual tumor activity seen by subtraction dynamic MRI and showed that 10 out of 16 (62.5\%) of hepatic focal lesions showing residual activity on subtraction dynamic MRI showed high signal on unenhanced T1 images. Our findings were contrasted by previous 
investigators who reported high $\mathrm{T} 1$ signal in only three out of 31 (9.7\%) of their unresolved lesions [19].

In the current study, we correlated the T2 signal intensity in the ablation zone and residual tumor activity. It was found that $63.6 \%$ of well-ablated hepatic focal lesions and $50 \%$ of lesions with residual disease showed intermediate T2 signals. No statistically significant difference between the T2 signal intensity and neoplastic viability according to subtraction MRI was found. The study findings were in agreement with previous investigators who found no significant correlation between T2 signal and neoplastic activity [20]. The current study results on the other hand were contrasted by Dromain and colleagues [15] who reported homogeneous hypointense T2 signal in $86 \%$ of their RFA treated lesions due to coagulative necrosis, while only $14 \%$ showed markedly high heterogeneous signal intensity due to liquefactive necrosis. The increased contrast between the viable remnant tumor, which has a high signal and the coagulated area, which has low signal, could explain T2 superior sensitivity. A study by Kierana and colleagues [18] concluded that areas of focal high T2 signal at the ablation margin are indicative of residual tumor activity and local tumor progression with a specificity of $100 \%$. Another study reported that focal nodular areas of eccentric T2 signal particularly when associated with enhancement is considered highly suspicious for residual or recurrent tumor activity [8]. Based on the results of the current study, combination of moderate hyperintense signal on T2-weighted images and equivalent enhancement on contrastenhanced T1-weighted images should provide high specificity for residual viable tumor following RFA. In this study, it was found that all the 14 hepatic focal lesions where there was disagreement between the first and second reader occurred in lesions having high signal in the pre-contrast T1 images similar to the results published by Winters and colleagues [14] who found that ablated zones show high signal on the unenhanced T1-weighted images can complicate the interpretation of the contrastenhanced MRI. In our experience, the high signal seen in contrast-enhanced T1-weighted images may be the result of enhancement, preexisting T1-weighted high signal or a combination of both.

Correlation between the findings of the first reader using conventional dynamic MRI and the second reader using subtraction dynamic MRI was performed with subtraction MRI considered the gold standard. Residual disease was reported by the first reader in 22 lesions, but the second reader disagreed in ten $(45.5 \%)$ of them where complete ablation was reported. Subtraction dynamic MRI also detected residual activity in four out of $38(10.5 \%)$ of well-ablated lesions reported by the first reader. Significant additive value of subtraction dynamic
MRI technique was observed in our study with a $P$ value of 0.009 indicating the importance of subtraction MRI in enhancing the reader confidence level in the evaluation of treatment response following RFA for HCC. Our findings were consistent with previous investigators who concluded that subtraction MRI is a useful problem-solving tool that significantly aids in the assessment of post-RFA treatment response by removing preexisting T1-weighted images high signal leaving only enhancement indicating residual tumor activity [4, 14]. Newatia et al. [12] concluded that subtraction MRI can enhance the subtle enhancement within the tumor making it more evident by removing the high $\mathrm{T} 1$ signal that is usually present due to coagulative tissue necrosis and can also differentiate between nodular peritumoral enhancement from peritumoral hyperemia which inevitably occurs following RFA.

Two out of 62 lesions from subtraction dynamic MRI imaging assessment were excluded due to misregistration. Similar findings were published by Winters et al. [14] with one out of 27 patients in their series being eliminated due to their inability to generate good quality subtraction images as a result of patient mobility between MRI acquisitions. Another study also concluded that the main problem in subtraction MR imaging was faulty coregistration between unenhanced and enhanced source images were [21]. Maintaining the patient position constant during the examination and using end expiration breath holds help to minimize this problem.

The limitation of this study is lack of histopathological confirmation as most patients who perform RFA do not undergo surgery. Therefore, pathologic confirmation was difficult to obtain. Furthermore, biopsy may result in sampling error as most recurrent or residual lesions are small. Another limitation is the small sample size. Further studies with larger sample size are needed in the future to further document and confirm the study findings.

\section{Conclusion}

MRI is a powerful imaging tool in the detection of tumor viability and complications after RFA in patients with HCC. Dynamic study is essential in detecting recurrent lesions, while subtraction technique is the gold standard for discriminating between the ablation zone's typical hyperintense T1 signal and arterial enhancement due to residual disease. The imaging protocol should include pre-contrast axial T1 TFE, axial T2 TSE and axial T2 SPAIR, dynamic study using THRIVE and post-processing subtraction images for better tissue characterization.

\section{Abbreviations}

CEUS: Contrast-enhanced ultrasound; CT: Computed tomography; DWI: Diffusion-weighted imaging; EASL: European Association for the Study of Liver Disease; FOV: Field of view; HCC: Hepatocellular carcinoma; MRI: Magnetic 
resonance imaging; RFA: Radiofrequency ablation; TFE: Turbo field echo; TSE: Turbo spin echo; SPAIR: Spectral attenuated inversion recovery; THRIVE: T1 high-resolution isotropic volume examination; TR: Repetition time; TE: Time to echo.

\section{Acknowledgements}

The authors would like to acknowledge MRI radiographers at National Cancer Institute who scanned the patients.

\section{Authors' contributions}

A.E.G involved in data collection and analysis, suggesting the idea, analysis and reporting MRI examinations, reviewing and editing the manuscript. H.S.W.M involved in data collection and analysis, analysis and reporting MRI examinations, reviewing and editing the manuscript. S.M.E. involved in review of literature, data collection and analysis, writing the original draft, reviewing and editing the manuscript. All authors have read and approved the manuscript.

\section{Funding}

The authors declare that they have did not receive funding for this research.

\section{Availability of data and materials}

The data sets used and analyzed during the current study are available from the corresponding author on reasonable request.

\section{Declarations}

\section{Ethics approval and consent to participate}

National Cancer Institute Ethics Committee approval was obtained. Reference number is not applicable. Written informed consent was obtained from all patients who agreed to take part in this study.

\section{Consent for publication}

All patients included in this research gave written informed consent to publish the data contained within the study.

\section{Competing interests}

The authors declare that they have no competing interests.

Received: 7 September 2021 Accepted: 21 November 2021

Published online: 26 November 2021

\section{References}

1. Ozkavukcu E, Haliloğlu N, Erden A (2009) Post-treatment MRI findings of hepatocellular carcinoma. Diagn Interv Radiol 15:111-120

2. Gaia S, Ciruolo M, Ribaldone DG et al (2021) Higher efficiency of percutaneous microwave (MWA) than radiofrequency ablation (RFA) in achieving complete response in cirrhotic patients with early hepatocellular carcinoma. Curr Oncol 28:1034-1044

3. Yoshida H, Taniai N, Yoshioka M et al (2019) Current status of laparoscopic hepatectomy. J Nippon Med Sch 86:201-206

4. Bréhier $G$, Besnier $L$, Delagnes A et al (2021) Imaging after percutaneous thermal and non-thermal ablation of hepatic tumour: normal appearances, progression and complications. Br J Radiol. https://doi.org/10. 1259/bjr.20201327

5. Bhardwaj N, Strickland AD, Ahmad F, Dennison AR, Lloyd DM (2010) Liver ablation techniques: a review. Surg Endosc 24:254-265

6. Kuehl H, Antoch G, Stergar H et al (2008) Comparison of FDG-PET, PET/ CT and MRI for follow-up of colorectal liver metastases treated with radiofrequency ablation: initial results. Eur J Radiol 67:362-371

7. Sainani NI, Gervais DA, Mueller PR, Arellano RS (2013) Imaging after percutaneous radiofrequency ablation of hepatic tumors: part 1, normal findings. Am J Roentgenol 200:184-193

8. Sainani NI, Gervais DA, Mueller PR, Arellano RS (2013) Imaging after percutaneous radiofrequency ablation of hepatic tumors: part 2, abnormal findings. Am J Roentgenol 200:194-204

9. European Association For The Study OfThe Liver; European Organization For Research And Treatment Of Cancer (2012) EASL-EORTC clinical practice guidelines: management of hepatocellular carcinoma. J Hepatol 56:908-943

10. Kele PG, van der Jagt EJ (2010) Diffusion weighted imaging in the liver. World J Gastroenterol 16:1567-1576

11. Thng CH, Koh TS, Collins DJ, Koh DM (2010) Perfusion magnetic resonance imaging of the liver. World J Gastroenterol 16:1598-1609

12. Newatia A, Khatri G, Friedman B, Hines J (2007) Subtraction imaging: applications for nonvascular abdominal MRI. Am J Roentgenol 188:1018-1025

13. Chopro S, Dodd GD 3rd, Chintapalli KN, Leyendecker JR, Karahan OI, Rhim H (2001) Tumor recurrence after radiofrequency thermal ablation of hepatic tumors: spectrum of findings on dual-phase contrast-enhanced CT. Am J Roentgenol 177:381-387

14. Winters SD, Jackson S, Armstrong GA, Birchall IW, Lee KH, Low G (2012) Value of subtraction MRI in assessing treatment response following image-guided loco-regional therapies for hepatocellular carcinoma. Clin Radiol 67:649-655

15. Dromain C, de Baere T, Elias D et al (2002) Hepatic tumors treated with percutaneous radio-frequency ablation: CT and MR imaging follow-up. Radiology 223:255-262

16. Hussain HK, Syed I, Nghiem HV et al (2004) T2-weighted MR imaging in the assessment of cirrhotic liver. Radiology 230:637-644

17. Limanond P, Zimmerman P, Raman SS, Kadell BM, Lu DS (2003) Interpretation of CT and MRI after radiofrequency ablation of hepatic malignancies. Am J Roentgenol 181:1635-1640

18. Kierans AS, Elazzazi M, Braga L et al (2010) Thermoablative treatments for malignant liver lesions: 10-year experience of MRI appearances of treatment response. Am J Roentgenol 194:523-529

19. Abddallah MFH, Desouky SHI, Madbouly M et al (2021) Follow-up after radiofrequency ablation of hepatocellular carcinoma: diffusion weighted and dynamic contrast enhanced MRI characteristics. Med J Cairo Univ 89:297-305

20. Vincenza G, Mario P, Roberta F et al (2013) Surveillance of HCC patients after liver RFA: role of MRI with hepatospecific contrast versus threephase CT scan-experience of high volume oncologic institute. Gastroenterolo Res Pract. https://doi.org/10.1155/2013/469097

21. Yu JS, Rofsky NM (2003) Dynamic subtraction MR imaging of the liver: advantages and pitfalls. Am J Roentgenol 180:1351-1357

\section{Publisher's Note}

Springer Nature remains neutral with regard to jurisdictional claims in published maps and institutional affiliations.

\section{Submit your manuscript to a SpringerOpen ${ }^{\circ}$ journal and benefit from:}

- Convenient online submission

- Rigorous peer review

- Open access: articles freely available online

- High visibility within the field

- Retaining the copyright to your article

Submit your next manuscript at springeropen.com 